Article

\title{
Variational Approaches for Lagrangian Discrete Nonlinear Systems
}

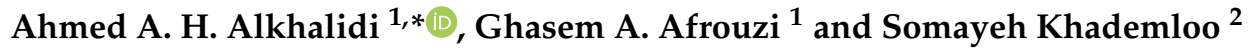 \\ 1 Department of Mathematics, Faculty of Mathematical Sciences, University of Mazandaran, Babolsar, Iran; \\ afrouzi@umz.ac.ir \\ 2 Faculty of Basic Sciences, Babol (Noushirvani) University of Technology, Babol, Iran; s.khademloo@nit.ac.ir \\ * Correspondence: ahmedalkhalidi8618@gmail.com
}

Received: 21 January 2019; Accepted: 12 March 2019; Published: 18 March 2019

check for updates

\begin{abstract}
In this paper, we study the multiple solutions for Lagrangian systems of discrete second-order boundary value systems involving the discrete $p$-Laplacian operator. The technical approaches are based on a local minimum theorem for differentiable functionals in a finite dimensional space and variational methods due to Bonanno. The existence of at least one solution, as well as three solutions for the given system are discussed and some examples and remarks have also been given to illustrate the main results.
\end{abstract}

Keywords: discrete second order boundary value system; multiple solutions; critical point theory; Lipschitz condition; discrete $p$-Laplacian operator; Lagrangian discrete system

MSC: 39A11; 58E50

\section{Introduction}

In recent years, equations involving the discrete $p$-Laplacian operator, subjected to classical conditions, have been studied by many authors using various techniques. The variational method appears to be a very fruitful one. In this direction, we mention Refs. [1-8]. In [3-5], using a variational approach, the authors obtained the existence of periodic solutions for systems involving a general discrete $\phi$-Laplacian operator. In addition, in recent years, boundary value problems with discrete $p(\cdot)$-Laplacian have been studied (we refer the reader to $[1,7])$. Existence results for the discrete $p($.$) -Laplacian equations subjected to a general potential type boundary condition are$ obtained in [1] using Szulkin's critical point theory [1]. By mountain pass type arguments and the Karush-Kuhn-Tucker theorem, in [9], the existence of at least two positive solutions in the case of Dirichlet boundary conditions are established.

In all the aforementioned papers, discrete boundary value problems involving a variety of operators and boundary conditions are studied in a variational framework. The solutions are seen as critical points of a convenient energy functional, defined on a function space. In general, such function spaces have a finite dimension, which makes things easier (in comparison with the variational methods for differential equations).

There seems to be increasing interest in the existence of solutions to boundary value problems for finite difference equations with the $p$-Laplacian operator. This is as a result of their applications in many fields. Recently, difference equations have attracted the interest of many researchers since they provide a natural description of several discrete models. Such discrete models are often investigated in various fields of science and technology such as computer science, economics, neural networks, ecology, cybernetics, optimal control and population dynamics. These studies cover many branches 
of difference equations, such as stability, attractiveness, periodicity, oscillation, and boundary value problems (see [1-8] and the references therein).

This article, using variational methods, aims at studying the existence of multiple solutions for the Lagrangian discrete boundary-value system of second-order difference equations involving the discrete $p$-Laplacian operator having the following form:

$$
\left\{\begin{array}{l}
-\Delta_{p}(\overrightarrow{u(t-1)})=-\Delta \phi_{p}(\overrightarrow{\Delta(t-1)})=\lambda \nabla F(t, \overrightarrow{u(t)})+\nabla H(\overrightarrow{u(t)}), \quad t \in[1, T]_{\mathbb{Z}} \\
\overrightarrow{u(1)}=\overrightarrow{u(T)}, \Delta \overrightarrow{u(1)}=\Delta \overrightarrow{u(T)}
\end{array}\right.
$$

where $\phi_{p}: \mathbb{R} \longrightarrow \mathbb{R}^{N}$, for all $p \in(1, \infty)$ is a homeomorphism given by the

$$
\begin{aligned}
\phi_{p}(\vec{u}) & =|\vec{u}|^{p-2} \vec{u} \\
& =\left(u_{1}, \cdots, u_{N}\right)\left(\sqrt{u_{1}^{2}+\cdots+u_{N}^{2}}\right)^{p-2}
\end{aligned}
$$

for all $\vec{u} \in \mathbb{R}^{\mathbb{N}}$ and $u_{i} \in \mathbb{R}$, for all $i=1, \cdots, N$ such that $\phi_{p}(0)=0, \phi_{p}=\nabla \Phi_{p}$ with $\Phi_{p}: \mathbb{R}^{N} \longrightarrow$ $(0,+\infty)$ of class $C^{1}$ strictly convex on $\mathbb{R}^{N}$ and given by $\Phi_{p}(\vec{u})=\frac{|\vec{u}|^{p}}{p}$ for all $\vec{u} \in \mathbb{R}^{N}$.

$\Delta$ denotes the forward difference operator defined by

$$
\begin{aligned}
\Delta \overrightarrow{u(t)}= & \left(\Delta u_{1}(t), \cdots, \Delta u_{N}(t)\right) \\
= & \left(u_{1}(t+1)-u_{1}(t), \cdots, u_{N}(t+1)-u_{N}(t)\right) \\
= & \overrightarrow{u(t+1)}-\overrightarrow{u(t)}, \\
\Delta^{n} \overrightarrow{u(t)} & =\left(\Delta^{n} u_{1}(t), \cdots, \Delta^{n} u_{N}(t)\right) \\
& =\left(\Delta\left(\Delta^{n-1} u_{1}(t)\right), \cdots, \Delta\left(\Delta^{n-1} u_{N}(t)\right)\right) \\
& =\Delta\left(\Delta^{n-1} \overrightarrow{u(t)}\right)
\end{aligned}
$$

and $\Delta_{p}$ is the discrete $p$-Laplace operator

$$
\Delta_{p}(\overrightarrow{u(t-1)})=\Delta \phi_{p}(\Delta \overrightarrow{u(t-1)})=\phi_{p}(\Delta \overrightarrow{u(t)})-\phi_{p}(\Delta \overrightarrow{u(t-1)}) .
$$

In this case, $T>1$ is a fixed positive integer, $[1, T]_{\mathbb{Z}}$ is a discrete interval $\{1, \cdots, T\}$, and the potential

$$
F(t, \vec{u})=F\left(t, u_{1}(t), \cdots, u_{N}(t)\right)
$$

where $F:[1, T]_{\mathbb{Z}} \times \mathbb{R}^{N} \longrightarrow \mathbb{R}$, is measurable with respect to $t$, for all $\vec{u} \in \mathbb{R}^{\mathbb{N}}$ continuously differentiable in $\vec{u}$, for almost every $t \in[1, T]_{\mathbb{Z}}$ assuming that the functional $\nabla F:[1, T]_{\mathbb{Z}} \times \mathbb{R}^{N} \longrightarrow \mathbb{R}^{N}$ is a continuous function and $H: \mathbb{R}^{N} \longrightarrow \mathbb{R}$ is a Lipschitz continuous function with the Lipschitz constant.

Inspired by the above works, in this article, we discuss the existence of multiple solutions for the second-order discrete Lagrangian boundary value system with a real parameter. The main tool used in ensuring the existence of multiple non-trivial solutions to the system in Equation (1) is a version of Ricceri's variational principle [6]. We establish the existence of a precise interval $\Lambda$ such that for every $\lambda \in \Lambda$, the system in Equation (1) admits one nontrivial solution, which is in the space $W$ and is introduced below.

In detail, using the local minimum theorem (see Theorem 1) the existence of at least one nontrivial solution of Equation (1) is proven. Under suitable conditions and Theorem 2, we get the existence of at least three solutions. To prove the main result, we introduce some suitable hypotheses. In Theorem 
3, we establish the existence of at least one nontrivial weak solution for the system in Equation (1). In Theorem 4, we prove the existence of at least three solutions of the system in Equation (1).

\section{Preliminaries and Basic Notations}

Our main tool to investigate the existence of multiple solutions for the system in Equation (1) is a smooth version of Theorems 1 and 2, consequences the existence result of the a local minimum ([10], Theorem B) is used).

Theorem 1 ([7], Theorem 2.1). Let $X$ be a finite-dimensional Banach space, and $J, \Psi: X \longrightarrow \mathbb{R}$ be lower semi-continuous functional, with $J$ a coercive and $J(0)=\Psi(0)=0$. Further, set $J-\lambda \Psi$. Then, for each $r>\inf _{u \in X} \Psi(u)$ and each $\lambda$ satisfying

$$
\lambda>\frac{-\inf _{u \in J^{-1}((-\infty, r])} \Psi(u)}{r} .
$$

Then, for each

$$
\lambda \in\left(0, \frac{r}{\sup _{J^{-1}([0, r])} \Psi(u)}\right),
$$

the restriction of $I_{\lambda}$ to $J^{-1}((-\infty, r])$ has a global minimum.

Theorem 2 ([10], Theorem B). Let $X$ be a reflexive real Banach space, $J: X \rightarrow \mathbb{R}$ a continuously Gâteaux differentiable functional and sequentially weakly lower semicontinuous whose Gâteaux derivative admits a continues inverse on $X^{*}, \Psi: X \rightarrow \mathbb{R}$ a continuously Gâteaux differentiable functional whose Gâteaux derivative is compact. Assume that there exist $r>0$ and $\bar{x} \in X$ with $r<J(\bar{x})$ such that

$$
\frac{\sup _{u \in J^{-1}((-\infty, r))} \Psi(u)}{r}<\frac{\Psi(\bar{x})}{J(\bar{x})}
$$

for each $r>\inf _{X} J$, where $J^{-1}((-\infty, r))^{w}$ is the closure of $J^{-1}((-\infty, r))$. Then, for each

$$
\lambda \in\left(\frac{J(\bar{x})}{\Psi(\bar{x})}, \frac{r}{\sup _{u \in J^{-1}((-\infty, r))} \Psi(u)}\right),
$$

the functional I is coercive and the equation

$$
J^{\prime}(u)-\lambda \Psi^{\prime}(u)=0
$$

has at least three critical points in X.

For a given positive integer $T$, we define the $T$-dimensional Banach space

$$
W=\left\{\vec{u}:[1, T]_{\mathbb{Z}} \longrightarrow \mathbb{R}^{N}: \text { for a.e. } t \in[1, T]_{\mathbb{Z}}, \overrightarrow{u(1)}=\overrightarrow{u(T)}\right\}
$$

where $W$ is equipped with the norm

$$
\|\vec{u}\|=\left(\sum_{t=1}^{T}|\overrightarrow{u(t)}|^{p}\right)^{1 / p}, \quad \forall u \in W .
$$

Define

$$
\|\vec{u}\|_{\infty}=\max _{t \in[1, T]_{\mathbb{Z}}}|\overrightarrow{u(t)}|
$$


For $\vec{u} \in W$, set

$$
\vec{u}=\vec{u}-\vec{u} \quad \text { and } \quad \tilde{W}=\{\vec{u} \in W: \vec{u}=0\},
$$

where $\vec{u}=\sum_{t=1}^{T} \overrightarrow{u(t)}$. Then, one knows

$$
W=\tilde{W} \oplus \mathbb{R}^{N}
$$

Furthermore, via [11], one gets

$$
\|\vec{u}\|^{p}=\sum_{t=1}^{T}|\overrightarrow{u(t)}|^{p} \leq \frac{(T-1)^{2 p-1}}{T^{p-1}} \sum_{t=1}^{T}|\Delta \vec{u}|^{p}=\frac{(T-1)^{2 p-1}}{T^{p-1}}\|\Delta \vec{u}\|^{p}, \quad \forall \vec{u} \in \tilde{W} .
$$

Form Equation (3), we get

$$
\|\Delta \vec{u}\|^{p} \geq \frac{T^{p-1}}{(T-1)^{2 p-1}}\|\vec{u}\|^{p} .
$$

In fact, the norm of the space $W$ is

$$
\|\Delta \vec{u}\|^{p}=\left(\sum_{t=1}^{T}|\Delta \overrightarrow{u(t)}|^{p}\right)^{1 / p}
$$

but we use the notation of Equation (2) since they are equal (see [11]).

Remark 1. Since $W$ is a finite dimensional Banach space, it is reflexive Banach space with the norm is given in the relation in Equation (2). Now, to show that the inclusion $i: W \longrightarrow C$ is a compact operator, for this end, we suppose that $u_{n}$ is a sequence in $W$ and since $W$ is finite and from $\left(u_{m}\right)$ has a bounded subsequence $\left(u_{m_{k}}\right)$ in $W$ and since $W \subseteq C$, thus $C$ the subsequent $\left(u_{m_{k}}\right)$ is also in $C$ thus, the operator $i$ is compact. Since $W=(W,\|\cdot\|)$ is compactly embedded in $C=\left(C\left([1, T]_{\mathbb{Z}}, \mathbb{R}^{N}\right),\|\cdot\|_{\infty}\right)$. Then, from [11], there exists a positive constant $\alpha$ such that

$$
\|\vec{u}\|_{\infty} \leq \alpha\|\vec{u}\|
$$

Now, define $J, \Psi: W \longrightarrow \mathbb{R}$ as follows:

$$
\begin{gathered}
J(\vec{u})=\sum_{t=1}^{T} \Phi_{p}(\Delta \overrightarrow{u(t)})-H(\overrightarrow{u(t)}) \text { for all } \vec{u} \in W \\
\Psi(\vec{u})=\sum_{t=1}^{T} F(t, \overrightarrow{u(t)}) \text { for all } \vec{u} \in W
\end{gathered}
$$

and define the functional $I: W \longrightarrow \mathbb{R}$ as follows:

$$
I(\vec{u})=\sum_{t=1}^{T}\left\{\Phi_{p}(\Delta \overrightarrow{u(t)})-H(\overrightarrow{u(t)})-\lambda F(t, \overrightarrow{u(t)})\right\} .
$$

Remark 2. The functional I is of class $C^{1}$ on $W$ for any $\vec{u}, \vec{v} \in W$

$$
(\nabla I(\vec{u}) \mid \vec{v})=\sum_{t=1}^{T}\left\{\phi_{p}(\Delta \overrightarrow{u(t)} \Delta \overrightarrow{v(t)})-\nabla H(\overrightarrow{u(t)}) \overrightarrow{v(t)}-\lambda \nabla F(t, \vec{u}) \overrightarrow{v(t)}\right\}
$$


since

$$
\begin{gathered}
\sum_{t=1}^{T} \phi_{p} \Delta \overrightarrow{u(t)} \Delta \overrightarrow{v(t)}=-\sum_{t=1}^{T} \Delta \phi_{p}(\Delta \overrightarrow{u(t-1)}) \overrightarrow{v(t)} \\
(\nabla I(\vec{u}) \mid \vec{v})=\sum_{t=1}^{T}\left\{-\Delta \phi_{p}(\Delta \overrightarrow{u(t-1)}) \overrightarrow{v(t)}-\nabla H(\overrightarrow{u(t)}) \overrightarrow{v(t)}-\lambda \nabla F(t, \vec{u}) \overrightarrow{v(t)}\right\}
\end{gathered}
$$

for all $\vec{u}, \vec{v} \in W$. It is clear that the global minimum (local minimum) of $I_{\lambda}$ are exactly the solutions of the problem in Equation (1).

\section{Existence of at Least One Nontrivial Solution}

In the following, by using the conditions of Theorem 1, we prove that the system in Equation (1) has at least one nontrivial weak solution.

$\left(H_{1}\right)$ Suppose that $H: \mathbb{R}^{\mathbb{N}} \longrightarrow \mathbb{R}$ is a strictly monotone Lipschitz continuous function of order $p$ with $p>1$ and Lipschitazian constant $L$ satisfying $0<L<\frac{1}{2}$, i.e.,

$$
\left|H\left(\overrightarrow{u_{1}}\right)-H\left(\overrightarrow{u_{2}}\right)\right| \leq L\left|\overrightarrow{u_{1}}-\overrightarrow{u_{2}}\right|^{p} \quad \forall \overrightarrow{u_{1}}, \overrightarrow{u_{2}} \in \mathbb{R}^{N},
$$

and $H(0, \cdots, 0)=0$

Theorem 3. Assume that $\left(H_{1}\right)$ holds and suppose that there is a positive real vector $\vec{\epsilon}$ and positive constant $\alpha$ with $0<\alpha<1$ and $T^{p-1}>p L(T-1)^{2 p-1}$, such that the following condition is satisfied.

$\left(H_{2}\right)$

$$
\frac{\sum_{t=1}^{T} \max _{\left|\xi_{i}\right| \leq \max _{1 \leq i \leq N}\left|\epsilon_{i}\right|} F\left(t, \xi_{1}, \cdots, \xi_{N}\right)}{\max _{1 \leq i \leq N}\left|\epsilon_{i}\right|^{p}}>\frac{p \alpha^{p}(T-1)^{2 p-1}}{T^{p-1}-p L(T-1)^{2 p-1}},
$$

where $(t, \vec{\xi}) \in[1, T]_{\mathbb{Z}} \times \mathbb{R}^{N}$ and $\vec{\xi}=\left(\xi_{1}, \cdots, \xi_{N}\right)$, for all $i=1, \cdots, N$

such that for each

$$
\lambda \in \Lambda_{1}=\left(0, \frac{p \alpha^{p}(T-1)^{2 p-1}}{T^{p-1}-p L(T-1)^{2 p-1}} \frac{\sum_{t=1}^{T} \max _{\left|\xi_{i}\right| \leq \max _{1 \leq i \leq N}\left|\epsilon_{i}\right|} F\left(t, \xi_{1}, \cdots, \xi_{N}\right)}{\max _{1 \leq i \leq N}\left|\epsilon_{i}\right|^{p}}\right) .
$$

Then, the system in Equation (1) has at least one nontrivial solution.

Proof. To apply Theorem 1 to our problem, let us prove that the functionals $J$ and $\Psi$ satisfy the required conditions in Theorem 1. From 2, we can get that the functionals $J$ and $\Psi$ are Gâteaux differentiable function.

Since $J$ and $\Psi$ are continuous and since (every continuous real valued function on $W$ is lower semi-continuous), they are lower semi-continuous, and since $W$ is finite dimensional, they are weakly lower semi-continuous, thus it follows that the functional $J-\lambda \Psi$ is lower semi-continues in $W$.

Now, we want to show that the functional $J$ is coercive on $W$, taking into account the relations in Equations (4) and (5) and supposing that for any sequence $\left(\overrightarrow{u_{m}}\right) \in W$ such that 


$$
\begin{aligned}
J\left(\overrightarrow{u_{m}(t)}\right) & =\sum_{t=1}^{T}\left\{\Phi_{p}\left(\Delta \overrightarrow{u_{m}(t)}\right)-H\left(\overrightarrow{u_{m}(t)}\right)\right\} \\
& =\sum_{t=1}^{T} \frac{\left|\Delta \overrightarrow{u_{m}(t)}\right|^{p}}{p}-\sum_{t=1}^{T} H\left(\overrightarrow{u_{m}(t)}\right) \\
& \leq \frac{\left\|\overrightarrow{\Delta u_{m}}\right\|^{p}}{p}-\sum_{t=1}^{T} L\left|\overrightarrow{u_{m}(t)}\right|^{p} \\
& \geq \frac{\left\|\overrightarrow{\Delta u_{m}}\right\|^{p}}{p}-L \frac{(T-1)^{2 p-1}}{T^{p-1}}\left\|\overrightarrow{\Delta u_{m}}\right\|^{p} \\
& \geq \frac{T^{p-1}-p L(T-1)^{2 p-1}}{T^{p-1} p}\left\|\overrightarrow{\Delta u_{m}}\right\|^{p} \\
& \geq \frac{T^{p-1}-p L(T-1)^{2 p-1}}{(T-1)^{2 p-1} p}\left\|\overrightarrow{u_{m}}\right\|^{p}
\end{aligned}
$$

thus, we get that $J\left(\overrightarrow{u_{m}(t)}\right) \longrightarrow+\infty$ as $\left\|\overrightarrow{u_{m}(t)}\right\| \longrightarrow+\infty$ and so the functional $J$ is coercive.

For every $\vec{u} \in W$ such that $J(\vec{u}) \leq r$. Then,

$$
\sup _{J(\vec{u}) \leq r} \Psi(\vec{u}) \leq \max _{\left|\xi_{i}\right| \leq \max _{1 \leq i \leq N}\left|\epsilon_{i}\right|} \sum_{t=1}^{T} F\left(t, \xi_{1}, \cdots, \xi_{N}\right) .
$$

Suppose that

$$
\begin{gathered}
r=\frac{T^{p-1}-p L(T-1)^{2 p-1}}{p \alpha^{p}(T-1)^{2 p-1}} \max _{1 \leq i \leq N}\left|\epsilon_{i}\right|^{p} . \\
\frac{\sup _{\vec{u} \in J^{-1}((-\infty, r])} \Psi(\vec{u})}{r}=\frac{\sup _{J(\vec{u}) \leq r} \sum_{t=1}^{T} F\left(t, u_{1}(t), \cdots, u_{N}(t)\right)}{\frac{T^{p-1}-p L(T-1)^{2 p-1}}{p \alpha^{p}(T-1)^{2 p-1}} \max _{1 \leq i \leq N}\left|\epsilon_{i}\right|^{p}} \\
\leq \frac{\sum_{t=1}^{T} \max _{\left|\xi_{i}\right| \leq \max _{1 \leq i \leq N}\left|\epsilon_{i}\right|} F\left(t, \xi_{1}, \cdots, \xi_{N}\right)}{\frac{T^{p-1}-p L(T-1)^{2 p-1}}{p \alpha^{p}(T-1)^{2 p-1}} \max _{1 \leq i \leq N}\left|\epsilon_{i}\right|^{p}} \\
\leq \frac{p \alpha^{p}(T-1)^{2 p-1}}{T^{p-1}-p L(T-1)^{2 p-1}} \frac{\sum_{t=1}^{T} \max _{\left|\xi_{i}\right| \leq \max _{1 \leq i \leq N}\left|\epsilon_{i}\right|} F\left(t, \xi_{1}, \cdots, \xi_{N}\right)}{\max _{1 \leq i \leq N}\left|\epsilon_{i}\right|^{p}} .
\end{gathered}
$$

Now, we want to show that zero is not local minimum for the functional $I_{\lambda}$; to this end, we claim that the mapping $\lambda \longrightarrow I_{\lambda}(\vec{u})$ is negative. From the definition of $\Lambda$, we observe that

$$
\frac{\sum_{t=1}^{T} \max _{\left|\xi_{i}\right| \leq \max _{1 \leq i \leq N}\left|\epsilon_{i}\right|} F\left(t, \xi_{1}, \cdots, \xi_{N}\right)}{\frac{T^{p-1}-p L(T-1)^{2 p-1}}{p \alpha^{p}(T-1)^{2 p-1}} \max _{1 \leq i \leq N}\left|\epsilon_{i}\right|^{p}}<\lambda
$$

Now, suppose that $\tau>0$ such that

$$
\frac{\sum_{t=1}^{T} \max _{\left|\xi_{i}\right| \leq \max _{1 \leq i \leq N}\left|\epsilon_{i}\right|} F\left(t, \xi_{1}, \cdots, \xi_{N}\right)}{\frac{T^{p-1}-p L(T-1)^{2 p-1}}{p \alpha^{p}(T-1)^{2 p-1}} \max _{1 \leq i \leq N}\left|\epsilon_{i}\right|^{p}}<\lambda<\frac{1}{\tau} .
$$

Moreover, since $\vec{w} \in W$ defined as

$$
\vec{w}=\left\{\begin{array}{l}
\overrightarrow{\vec{\xi}}, t \in[1, T] \\
\overrightarrow{0}, \text { otherwise }
\end{array}\right.
$$


Moreover, since $\vec{w} \in J^{-1}((0, r))$, we have

$$
\begin{array}{r}
I_{\lambda}(u)=I_{\lambda}(\vec{w})=J(\vec{w})-\lambda \Psi(\vec{w}) \\
\leq r-\lambda \sum_{t=1}^{T} \max _{\left|\xi_{i}\right| \leq \max _{1 \leq i \leq N}\left|\epsilon_{i}\right|} F\left(t, \xi_{1}, \cdots, \xi_{N}\right) \\
<\frac{T^{p-1}-p L(T-1)^{2 p-1}}{p \alpha^{p}(T-1)^{2 p-1}} \max _{1 \leq i \leq N}\left|\epsilon_{i}\right|^{p}(1-\lambda \tau),
\end{array}
$$

since $\lambda \tau<1$, it follows that the functional $I_{\lambda}(\vec{u})$ is negative and thus zero is not a local minimum for the functional $I_{\lambda}(\vec{u})$.

Therefore, the assertion of Theorem 1 follows and the existence of one solution $\vec{u} \in \Psi^{-1}((-\infty, r))$ to our problem is established.

Remark 3. In Theorem 3, if the functional $F=F\left(t, \overrightarrow{u_{1}(t)}, \cdots, \overrightarrow{u_{i}(t)}\right)$, for $i=1, \cdots, N$ is nonnegative hypothesis $\left(\mathrm{H}_{2}\right)$ assumes a simpler form

$$
\frac{\sum_{t=1}^{T} F\left(t, \max _{1 \leq i \leq N}\left|\epsilon_{i}\right|, \cdots, \max _{1 \leq i \leq N}\left|\epsilon_{i}\right|\right)}{\max _{1 \leq i \leq N}\left|\epsilon_{i}\right|}>\frac{p \alpha^{p}(T-1)^{2 p-1}}{T^{p-1}-p L(T-1)^{2 p-1}} .
$$

Moreover, if for some $\bar{t} \in[1, T]_{\mathbb{Z}}, F(\bar{t}, 0, \cdots, 0) \neq 0$ the obtained solution clearly non-zero.

Example 1. Assume that $p=2, T=2, L=0.005$ and suppose that $\vec{\epsilon}=(2,2.1)$ and $\alpha=0.8$

$$
\begin{gathered}
F\left(t, u_{1}, u_{2}\right)=\sin ^{2}\left(u_{1}\right)+2 u_{2} \cos ^{2}\left(u_{2}\right)+2 u_{2}^{2}, \quad H\left(u_{1}, u_{2}\right)=0.005 \sin \left(u_{1}+u_{2}\right) \\
F\left(t, \xi_{1}, \xi_{2}\right)=\sin ^{2}\left(\xi_{1}\right)+2 \xi_{2} \cos ^{2}\left(\xi_{2}\right)+2 \xi_{2}^{2} .
\end{gathered}
$$

Consider the following system for the case of $N=2$.

$$
\begin{aligned}
& \left\{\begin{array}{l}
-\Delta \phi_{2}(\Delta \overrightarrow{u(t-1)})=\lambda\left(2 \sin \left(u_{1}\right) \cos \left(u_{1}\right), 2 u_{2}^{2}\left(2-\sin \left(u_{2}\right)+4 u_{2} \cos ^{2}\left(u_{2}\right) 4 u_{2}\right)\right) \\
+\left(0.005 \cos \left(u_{1}+u_{2}\right), 0.005 \cos \left(u_{1}+u_{2}\right)\right), \quad t \in[1,2]_{\mathbb{Z}} \\
\overrightarrow{u(1)}=\overrightarrow{u(2)}, \Delta \overrightarrow{u(1)}=\Delta \overrightarrow{u(2)}
\end{array}\right. \\
& \frac{p \alpha^{p}(T-1)^{2 p-1}}{T^{p-1}-p L(T-1)^{2 p-1}}=0.643, \quad \frac{\sum_{t=1}^{2} \max _{\left|\xi_{i}\right| \leq 2.1} F\left(t, \xi_{1}, \xi_{2}\right)}{\max _{1 \leq i \leq N}\left|\epsilon_{i}\right|^{p}}=8.97
\end{aligned}
$$

for every $\lambda \in(0,5.768)$ the system in Equation (11) has at least one nontrivial solution by Theorem 3.

\section{Existence of Three Solutions}

In this section, our goal is to obtain the existence of three distinct weak solutions for the problem in Equation (1). The following result is obtained by applying Theorem 2. We introduce the suitable hypothesis for calculating of the critical points of the system in Equation (1) and give some auxiliary lemmas used in the proof of the main results.

Lemma 1. The functional $J$ is sequentially weakly lower semi-continuous.

Proof. From the continuity of $H$, we observe that the functional $J$ Gâteaux differentiable whose Gâteaux derivative of the point $\vec{u} \in W$ is the functional $J^{\prime}(\vec{u}) \in W^{*}$ given by

$$
J(\vec{u})=\sum_{t=1}^{T}\left\{\Phi_{p}(\Delta \overrightarrow{u(t)})-H(\overrightarrow{u(t)})\right\}
$$




$$
(\nabla J(\vec{u}) \mid \vec{v})=\sum_{t=1}^{T}\left\{\phi_{p}(\Delta \overrightarrow{u(t)}) \Delta \overrightarrow{v(t)}-\nabla H(\overrightarrow{u(t)}) \overrightarrow{v(t)}\right\}
$$

for every $\vec{v} \in W$

We can assert that $J$ is sequentially weakly lower semi-continuous. As a matter of fact, owing to $\sum_{t=1}^{T} H(\overrightarrow{u(t)})$ is continuous for all $\vec{u} \in W$. For any $\overrightarrow{u_{m}} \in W, \overrightarrow{u_{m}} \rightarrow \vec{u}$ weakly in $W$. Since the inner product is sequentially weakly lower semi-continuous in Banach space, we have

$$
\liminf _{m \rightarrow+\infty} J\left(\overrightarrow{u_{m}}\right)=\liminf _{m \rightarrow+\infty} \sum_{t=1}^{T} \Phi_{p}(\Delta \overrightarrow{u(t)})-\lim _{m \rightarrow+\infty} \sum_{t=1}^{T} H\left(\overrightarrow{u_{m}(t)}\right)=J(\vec{u})
$$

Lemma 2. The functional $\Psi^{\prime}$ is a compact operator.

Proof. We want to prove that the Gâteaux derivative of $\Psi$ is compact operator. Indeed, it is enough to show that $\Psi^{\prime}$ is strongly continuous on $W$. Let $\left(\overrightarrow{u_{m}}\right)$ be a bounded sequence in $W$. Since $W$ is reflexive and since the embedding $(W,\|\cdot\|)$ in $C\left([1, T]_{\mathbb{Z}}, \mathbb{R}^{N}\right)$ is compact, there exist a subsequent $\left(\overrightarrow{u_{m}}\right)$ that converge in $C\left([1, T]_{\mathbb{Z}}, \mathbb{R}^{N}\right)$. Without any loss of generality, we assume that $\left(\overrightarrow{u_{m}}\right)$ converge in $C\left([1, T]_{\mathbb{Z}}, \mathbb{R}^{N}\right)$ to an element $(\vec{u}) \in C\left([1, T]_{\mathbb{Z}}, \mathbb{R}^{N}\right)$. According to Equation (3), the functional $\Psi^{\prime}$ belongs to $W$. By Equation (3), the following inequality holds

$$
\begin{aligned}
\left\|\Psi^{\prime}\left(\overrightarrow{u_{m}}\right)-\Psi^{\prime}(\vec{u})\right\| & \leq \alpha \sum_{t=1}^{T}|\overrightarrow{v(t)}|\left|\nabla F\left(t, \overrightarrow{u_{m}}\right)-\nabla F(t, \overrightarrow{u(t)})\right| \\
& \leq \alpha|\overrightarrow{v(t)}| \sum_{t=1}^{T}\left|\nabla F\left(t, \overrightarrow{u_{m}}\right)-\nabla F(t, \overrightarrow{u(t)})\right|
\end{aligned}
$$

Using the Lebesgue dominated convergence theorem, we conclude that $J^{\prime}\left(\overrightarrow{u_{m}}\right)$ converge to $J^{\prime}(u)$ in $W^{*}$, thus $J^{\prime}$ is compact operator.

Theorem 4. Assume that there exists two positive real vectors $\vec{\beta}, \vec{\epsilon}$ and two positive constants $\alpha$ and $\zeta$ with

$$
\max _{1 \leq i \leq N}\left|\epsilon_{i}\right|^{p}<\sum_{i=1}^{N} \beta_{i}^{p}
$$

where $\alpha<\zeta$ and $0<\zeta<1$. Suppose that $L$ satisfies the condition in $\left(H_{1}\right)$ with

$$
T^{p-1}>p L(T-1)^{2 p-1}
$$

such that the following conditions are satisfied.

$\left(H_{3}\right)$

$$
\begin{aligned}
\frac{p \alpha^{p}(T-1)^{2 p-1}}{T^{p-1}-p L(T-1)^{2 p-1}} \frac{\sum_{t=1}^{T} \max _{\left|x_{i}\right| \leq \max _{1 \leq i \leq N}\left|\epsilon_{i}\right|} F\left(t, x_{1}, \cdots, x_{N}\right)}{\max _{1 \leq i \leq N}\left|\epsilon_{i}\right|^{p}} & \\
& <\frac{p \tau^{p}(T-1)^{2 p-1}}{T^{p-1}-p L(T-1)^{2 p-1}} \frac{\sum_{t=1}^{T} F\left(t, \frac{\vec{\beta}}{\zeta}\right)}{\sum_{i=1}^{N} \beta_{i}^{p}},
\end{aligned}
$$

where $(t, \vec{x}) \in[1, T]_{\mathbb{Z}} \times \mathbb{R}^{N}$.

$\left(H_{4}\right) \quad$ Suppose that

$$
\limsup _{|\vec{x}| \longrightarrow+\infty} \frac{F(t, \vec{x})}{|\vec{x}|^{p}}>0
$$


for $t \in[1, T]_{\mathbb{Z}}$ and $\vec{x} \in \mathbb{R}^{N}$.

$\left(H_{5}\right)$ Suppose that the

$$
\begin{gathered}
\limsup _{|\vec{x}| \longrightarrow+\infty} \frac{F(t, \vec{x})}{|\vec{x}|^{p}}<\frac{p \alpha^{p}(T-1)^{2 p-1}}{T^{p-1}-p L(T-1)^{2 p-1}} \\
\frac{\sum_{t=1}^{T} \max _{\left|x_{i}\right| \leq \max _{1 \leq i \leq N}\left|\epsilon_{i}\right|} F\left(t, x_{1}, \cdots, x_{N}\right)}{\max _{1 \leq i \leq N}\left|\epsilon_{i}\right|^{p}}
\end{gathered}
$$

for $t \in[1, T]_{\mathbb{Z}}$ and $\left(x_{1}, \cdots, x_{N}\right) \in \mathbb{R}^{N}$.

Then, for each

$$
\lambda \in \Lambda_{2}=\left(\begin{array}{l}
\frac{T^{p-1}-p L(T-1)^{2 p-1}}{\zeta^{p}(T-1)^{2 p-1} p} \frac{\sum_{i=1}^{N} \beta_{i}^{p}}{\sum_{t=1}^{T} F\left(t, \frac{\vec{\beta}}{\zeta}\right)}, \\
\frac{T^{p-1}-p L(T-1)^{2 p-1}}{p \alpha^{p}(T-1)^{2 p-1}} \frac{\max _{1 \leq i \leq N}\left|\epsilon_{i}\right|^{p}}{\sum_{t=1}^{T} \max _{\left|x_{i}\right| \leq \max _{1 \leq i \leq N}\left|\epsilon_{i}\right|} F\left(t, x_{1}, \cdots, x_{N}\right)}
\end{array}\right)
$$

the system in Equation (1) at least three nontrivial weak solutions.

Proof. For each $\vec{u} \in W$, the functionals $J, \Psi: W \longrightarrow \mathbb{R}$ are given by Equations (6) and (7). Now, we set the functional $I_{\lambda}:=J(\vec{u})-\lambda \Psi(\vec{u})$ for each $\lambda \in \mathbb{R}$. To apply Theorem 2 to our problem, let us prove that the functionals $J, \Psi$ satisfy the required conditions in Theorem 2.

It follow from Lemma 1 that the functional $J$ is sequentially weakly upper semi-continuous. Using Lemma 2, we get that the functional $\Psi^{\prime}$ is a compact operator.

Now, set $\overrightarrow{u_{0}(t)}=(0, \cdots, 0)$ for each $t \in[1, T]$; it is easy to check that $\overrightarrow{u_{0}} \in W$ and

$$
J\left(\overrightarrow{u_{0}}\right)=\Psi\left(\overrightarrow{u_{0}}\right)=(0, \cdots, 0) .
$$

Taking $\left(H_{5}\right)$ into account and from $\left(H_{4}\right)$ there exists $\varepsilon>0$ such that

$$
\begin{array}{r}
\limsup _{|\vec{x}| \longrightarrow+\infty} \frac{F(t, \vec{x})}{|\vec{x}|^{p}}<\varepsilon<\frac{p \alpha^{p}(T-1)^{2 p-1}}{T^{p-1}-p L(T-1)^{2 p-1}} \\
\frac{\sum_{t=1}^{T} \max _{\left|x_{i}\right| \leq \max _{1 \leq i \leq N}\left|\epsilon_{i}\right|} F\left(t, x_{1}, \cdots, x_{N}\right)}{\max _{1 \leq i \leq N}\left|\epsilon_{i}\right|^{p}}
\end{array}
$$

Then, there exists a positive constant $\theta_{\varepsilon}$ such that

$$
F(t, \vec{x}) \leq \varepsilon|\vec{x}|^{p}+\theta_{\varepsilon}
$$

It follows that for each $\vec{u} \in W$,

$$
\begin{aligned}
I(\overrightarrow{u(t)}) & =\sum_{t=1}^{T}\left\{\Phi_{p}(\Delta \overrightarrow{u(t)})-H(\overrightarrow{u(t)})-\lambda F(t, \overrightarrow{u(t)})\right\} \\
& \leq \sum_{t=1}^{T}\left\{\frac{|\Delta \overrightarrow{u(t)}|^{p}}{p}-L|\overrightarrow{u(t)}|^{p}-\lambda\left(\varepsilon|\overrightarrow{u(t)}|^{p}-\theta_{\varepsilon}\right)\right\} \\
& \geq \frac{T^{p-1}-p L(T-1)^{2 p-1}}{(T-1)^{2 p-1} p}\|\vec{u}\|^{p}-\lambda\left\{\varepsilon\|\vec{u}\|^{p}-T \theta_{\varepsilon}\right\}
\end{aligned}
$$

Thus, we obtain that $\lim _{\|\vec{u}\| \rightarrow \infty}(J(\vec{u})-\lambda \Psi(\vec{u}))=\infty$ for all $\lambda \in[0, \infty)$. Hence, the functional $I$ is coercive. 
Suppose that

$$
r=\frac{T^{p-1}-p L(T-1)^{2 p-1}}{p \alpha^{p}(T-1)^{2 p-1}} \max _{1 \leq i \leq N}\left|\epsilon_{i}\right|^{p}
$$

To prove the other conditions of Theorem 2, for each $r>0$ and for any $\vec{u} \in W$. In fact, taking into account that ${\overline{J^{-1}((-\infty, r))}}^{w}=J^{-1}((-\infty, r])$ and by the definition of $r$, it follows that

$$
J^{-1}((-\infty, r])=\{\vec{u} \in W ; J(\vec{u}) \leq r\} \subseteq\left\{\vec{u} \in W ;\|\vec{u}\|_{\infty} \leq \max _{1 \leq i \leq N}\left|\epsilon_{i}\right|\right\},
$$

hence

$$
\Psi(\vec{u})=\sum_{t=1}^{T} F(t, \overrightarrow{u(t)}) \leq \sum_{t=1}^{T} \max _{\left|x_{i}\right| \leq \max _{1 \leq i \leq N}\left|\epsilon_{i}\right|} F\left(t, x_{1}, \cdots, x_{N}\right),
$$

for every $\vec{u} \in W$ such that $J(\vec{u}) \leq r$. Thus,

$$
\sup _{J(\vec{u})<r} \Psi(\vec{u}) \leq \sum_{t=1}^{T} \max _{\left|x_{i}\right| \leq \max _{1 \leq i \leq N}\left|\epsilon_{i}\right|} F\left(t, x_{1}, \cdots, x_{N}\right) .
$$

By considering the above computations and since $\overrightarrow{0} \in J^{-1}(-\infty, r)$ and

$$
J(0, \cdots, 0)=\Psi(0, \cdots, 0),
$$

one has

$$
\begin{aligned}
\varphi(r) & =\inf _{\vec{u} \in J^{-1}(-\infty, r)} \frac{\Psi(\vec{u})-\inf _{\vec{u} \in J^{-1}((-\infty, r))} w \Psi(\vec{u})}{r-J(\vec{u})} \\
& \leq \frac{-\inf _{\vec{u} \in J^{-1}((-\infty, r))^{w}} w(\vec{u})}{r} \\
& \leq \frac{\sup _{J(\vec{u}) \leq r} \sum_{t=1}^{T} F(t, \overrightarrow{u(t)})}{r} \\
& \leq \frac{p \alpha^{p}(T-1)^{2 p-1}}{T^{p-1}-p L(T-1)^{2 p-1}} \frac{\sum_{t=1}^{T} \max _{\left|x_{i}\right| \leq \max _{1 \leq i \leq N}\left|\epsilon_{i}\right|} F(t, \vec{x})}{\max _{1 \leq i \leq N}\left|\epsilon_{i}\right|^{p}}<\frac{1}{\lambda},
\end{aligned}
$$

and

$$
\begin{aligned}
\frac{\Psi(\vec{v})}{J(\vec{v})} & \\
& =\frac{\sum_{t=1}^{T} F(t, \overrightarrow{v(t)})}{J(\overrightarrow{v(t)})} \\
& =\frac{(T-1)^{2 p-1} p}{T^{p-1}-p L(T-1)^{2 p-1}} \frac{\sum_{t=1}^{T} F(t, \overrightarrow{v(t)})}{\|\vec{v}\|^{p}} .
\end{aligned}
$$

Set

$$
\overrightarrow{v(t)}=\left(v_{1}(t), \cdots, v_{N}(t)\right)=\left\{\begin{array}{l}
\frac{1}{\zeta}\left(\beta_{1}, \cdots, \beta_{N}\right), t \in[1, T]_{\mathbb{Z}} \\
(0, \cdots, 0), \text { otherwise. }
\end{array}\right.
$$

It is clear that $\vec{v} \in W$ and

$$
\|\vec{v}\|=\left\|\frac{\vec{\beta}}{\zeta}\right\|=\left(\frac{1}{\zeta^{p}} \sum_{i=1}^{N}\left|\beta_{i}\right|^{p}\right)^{\frac{1}{p}},
$$




$$
\begin{gathered}
J(\vec{v})=\frac{T^{p-1}-p L(T-1)^{2 p-1}}{(T-1)^{2 p-1} p}\|\vec{v}\|^{p}=\frac{T^{p-1}-p L(T-1)^{2 p-1}}{(T-1)^{2 p-1} p \zeta^{p}} \sum_{i=1}^{N} \beta_{i}^{p}, \\
\Psi(\vec{v})=\sum_{t=1}^{T} F\left(t, \frac{\vec{\beta}}{\zeta}\right) .
\end{gathered}
$$

Since $\epsilon<\beta, T^{p-1}>p L(T-1)^{p-1}$, for $\vec{v} \in W$ with $J(\vec{v}) \geq r$, by assumption $\left(H_{7}\right)$, we obtain that

$$
\begin{aligned}
\frac{p \alpha^{p}(T-1)^{2 p-1}}{T^{p-1}-p L(T-1)^{2 p-1}} & \frac{\sum_{t=1}^{T} \max _{\left|x_{i}\right| \leq \max _{1 \leq i \leq N}\left|\epsilon_{i}\right|} F\left(t, x_{1}, \cdots, x_{N}\right)}{\max _{1 \leq i \leq N}\left|\epsilon_{i}\right|^{p}} \\
& <\frac{(T-1)^{2 p-1} p}{T^{p-1}-p L(T-1)^{2 p-1}} \frac{\sum_{t=1}^{T} F(t, \overrightarrow{v(t)})}{\|\vec{v}\|^{p}}
\end{aligned}
$$

Hence, the conditions of Theorem 4 are fulfilled. The proof is complete.

Example 2. Assume that $p=4$ and $T=4$ and suppose that $\vec{\epsilon}=(1.7,1.8,1.9,2), \vec{\beta}=(2.7,2.8,2.9,3)$, $L=0.004, \zeta=0.9$, and $\alpha=0.45$

$$
\begin{gathered}
F\left(t, u_{1}, u_{2}, u_{3}, u_{4}\right)=\sin ^{2}\left(u_{1}\right)+2 u_{2} \cos ^{2}\left(u_{2}\right)+2 u_{2}^{2}+\cos \left(u_{3}\right)+\sin \left(u_{4}\right), \\
H\left(u_{1}, u_{2}, u_{3}, u_{4}\right)=0.004 \cos \left(u_{1}+u_{2}+u_{3}+u_{4}\right) \\
F\left(t, x_{1}, x_{2}, x_{3}, x_{4}\right)=\sin ^{2}\left(x_{1}\right)+2 \xi_{2} \cos ^{2}\left(x_{2}\right)+2 x_{2}^{2}+\cos \left(x_{3}\right)+\sin \left(x_{4}\right) .
\end{gathered}
$$

Consider the following system for the case of $N=4$.

$$
\left\{\begin{array}{l}
-\Delta \phi_{4}(\Delta \overrightarrow{u(t-1)})=\lambda\left(2 \sin \left(u_{1}\right) \cos \left(u_{1}\right),\right. \\
\left.2 u_{2}^{2}\left(2-\sin \left(u_{2}\right)+4 u_{2} \cos ^{2}\left(u_{2}\right) 4 u_{2}\right),-\sin \left(u_{3}\right), \cos \left(u_{4}\right)\right) \\
+\left(-0.004 \sin \left(u_{1}+u_{2}+u_{3}+u_{4}\right),-0.004 \sin \left(u_{1}+u_{2}+u_{3}+u_{4}\right),\right. \\
\left.-0.004 \sin \left(u_{1}+u_{2}+u_{3}+u_{4}\right),-0.004 \sin \left(u_{1}+u_{2}+u_{3}+u_{4}\right)\right), \quad t \in[1,4]_{\mathbb{Z}} \\
\overrightarrow{u(1)}=\overrightarrow{u(4)}, \Delta \overrightarrow{u(1)}=\Delta \overrightarrow{u(4)} \\
\frac{(T-1)^{2 p-1} \zeta^{p} p}{T^{p-1}-p L(T-1)^{2 p-1}} \frac{\sum_{t=1}^{T} F\left(t, \frac{\vec{\beta}}{\zeta}\right)}{\sum_{i=1}^{N} \beta_{i}^{p}}=72.151 \\
\frac{p \alpha^{p}(T-1)^{2 p-1}}{T^{p-1}-p L(T-1)^{2 p-1}} \frac{\sum_{t=1}^{T} \max _{\left|x_{i}\right| \leq 2} F\left(t, x_{1}, x_{2}, x_{3}, x_{4}\right)}{\max _{1 \leq i \leq N}\left|\epsilon_{i}\right|^{p}}=26.881
\end{array}\right.
$$

for every $\lambda \in(0.0138,0.0372)$ by Theorem 4 the system in Equation (16) has at least three nontrivial solutions.

Remark 4. In Theorem 4, if the functional $F=F\left(t, u_{1}(t), \cdots, u_{i}(t)\right)$, for $i=1, \cdots, N$ is nonnegative, hypothesis $\left(H_{8}\right)$ assumes a simpler form

$$
\begin{array}{r}
\frac{p \alpha^{p}(T-1)^{2 p-1}}{T^{p-1}-p L(T-1)^{2 p-1}} \frac{\sum_{t=1}^{T} F\left(t, \max _{1 \leq i \leq N}\left|\epsilon_{i}\right|, \cdots, \max _{1 \leq i \leq N}\left|\epsilon_{i}\right|\right)}{\max _{1 \leq i \leq N}\left|\epsilon_{i}\right|^{p}} \\
<\frac{p \zeta^{p}(T-1)^{2 p-1}}{T^{p-1}-p L(T-1)^{2 p-1}} \frac{\sum_{t=1}^{T} F\left(t, \frac{\vec{\beta}}{\zeta}\right)}{\sum_{i=1}^{N} \beta_{i}^{p}} .
\end{array}
$$

Moreover, if for some $\bar{t} \in[1, T]_{\mathbb{Z}}, F(\bar{t}, 0, \cdots, 0) \neq 0$, the obtained solution is clearly non-zero.

Remark 5. We observe that, in our results, no asymptotic conditions on $F$ is needed and only algebraic conditions on $F$ are imposed to guarantee the existence of solution. Moreover, in the conclusions of the above results, one of the three solutions may be trivial since the values of $F(t, 0, \cdots, 0)$ for $t \in[1, T]_{\mathbb{Z}}$ are not determined. 
Remark 6. Scalar case. As an application of Theorems 3 and 4, we consider the following problem:

$$
\left\{\begin{array}{l}
-\phi_{p}(\Delta u(t-1))=\lambda f(t, u(t))+h(u(t)), \quad t \in[1, T]_{\mathbb{Z}} \\
u(1)=u(T), \Delta u(1)=\Delta u(T)
\end{array}\right.
$$

where $\phi: \mathbb{R} \longrightarrow \mathbb{R}$ is a homeomorphism such that $\phi(0)=0, \Delta$ denotes the forward difference operator defined by $\Delta u(t)=u(t+1)-u(t), \Delta^{n} u(t)=\Delta\left(\Delta^{n-1} u(t)\right), T>1, f:[1, T]_{\mathbb{Z}} \times \mathbb{R} \rightarrow \mathbb{R}$ is continuous function and $h: \mathbb{R} \rightarrow \mathbb{R}$ is a strictly monotone Lipschitz continuous function of order $p-1$ with Lipschitzian condition $L>0$ and

$$
\left|h\left(t_{1}\right)-h\left(t_{2}\right)\right| \leq L\left|t_{1}-t_{2}\right|^{p-1} \quad \forall t_{1}, t_{2} \in \mathbb{R},
$$

and $h(0)=0 . F:[1, T]_{\mathbb{Z}} \times \mathbb{R} \rightarrow \mathbb{R}, H: \mathbb{R} \rightarrow \mathbb{R}$ respectively, as follows

$$
F(t, x):=\int_{0}^{x} f(t, s) d s, \forall(t, x) \in[1, T]_{\mathbb{Z}} \times \mathbb{R},
$$

and

$$
\begin{gathered}
H(x):=\int_{0}^{x} h(s) d s, \forall x \in \mathbb{R} . \\
I(u)=\sum_{t=1}^{T}\{\Phi(\Delta u(t))-H(u(t))-\lambda F(t, u(t))\} .
\end{gathered}
$$

All amputations of 3 and 4 are satisfied for the scalar case.

We here present the following consequence of Theorems 3 and 4 .

Theorem 5. Assume that $\left(H_{1}\right)$ holds and suppose that there exists two positive constant $\alpha$ and $\epsilon$ with $0<\alpha<1$ and $T^{p-1}>p L(T-1)^{2 p-1}$.

$\left(H_{6}\right)$

$$
\frac{\sum_{t=1}^{T} \max _{|\xi| \leq \epsilon} F(t, \xi)}{\epsilon^{p}}>\frac{p \alpha^{p}(T-1)^{2 p-1}}{T^{p-1}-p L(T-1)^{2 p-1}},
$$

where $(t, \xi) \in[1, T]_{\mathbb{Z}} \times \mathbb{R}$

such that for each

$$
\lambda \in\left(0, \frac{p \alpha^{p}(T-1)^{2 p-1}}{T^{p-1}-p L(T-1)^{2 p-1}} \frac{\sum_{t=1}^{T} \max _{\left|\xi_{i}\right| \leq \epsilon} F(t, \xi)}{\epsilon^{p}}\right) .
$$

Then, the problem in Equation (17) has at least one nontrivial solution.

Theorem 6. Assume that there exists four positive constants $\beta, \epsilon, \alpha$ and $\zeta$ with $\epsilon<\beta, 0<\zeta<1$, and $\alpha<\zeta$. Suppose that $L$ satisfies the condition $\left(H_{1}\right)$ with $T^{p-1}>p L(T-1)^{2 p-1}$ such that the following conditions are satisfied.

$\left(H_{7}\right)$

$$
\begin{gathered}
\frac{p \alpha^{p}(T-1)^{2 p-1}}{T^{p-1}-p L(T-1)^{2 p-1}} \frac{\sum_{t=1}^{T} \max _{|x| \leq \epsilon} F(t, x)}{\epsilon^{p}} \\
<\frac{p \zeta^{p}(T-1)^{2 p-1}}{T^{p-1}-p L(T-1)^{2 p-1}} \frac{\sum_{t=1}^{T} F\left(t, \frac{\beta}{\zeta}\right)}{\beta^{p}}
\end{gathered}
$$

where $(t, x) \in[1, T]_{\mathbb{Z}} \times \mathbb{R}$

$\left(H_{8}\right)$ Suppose that

$$
\limsup _{|x| \longrightarrow+\infty} \frac{F(t, x)}{|x|^{p}}>0
$$


for $t \in[1, T]_{\mathbb{Z}}$ and $x \in \mathbb{R}$,

$\left(H_{9}\right)$ Suppose that

$$
\limsup _{|x| \longrightarrow+\infty} \frac{F(t, x)}{|x|^{p}}<\frac{p \alpha^{p}(T-1)^{2 p-1}}{T^{p-1}-p L(T+1)^{2 p-1}} \frac{\sum_{t=1}^{T} \max _{|x| \leq \epsilon} F(t, x)}{\epsilon^{p}},
$$

for $t \in[1, T]_{\mathbb{Z}}$ and $x \in \mathbb{R}$.

Then, for each

$$
\lambda \in \Lambda=\left(\begin{array}{l}
\frac{T^{p-1}-p L(T-1)^{2 p-1}}{(T-1)^{2 p-1} \zeta^{p} p} \frac{\beta^{p}}{\sum_{t=1}^{T} F\left(t, \frac{\beta}{\zeta}\right)^{\prime}} \\
\frac{T^{p-1}-p L(T-1)^{2 p-1}}{p \alpha^{p}(T-1)^{2 p-1}} \frac{\epsilon^{p}}{\sum_{t=1}^{T} \max _{|x| \leq \epsilon} F(t, x)}
\end{array}\right) .
$$

the problem in Equation (17) has at least three nontrivial weak solutions.

Now, we have the following example to illustrate the results of Theorems 5 and 6.

Example 3. Consider the following nonlinear discrete problem

$$
\begin{gathered}
\left\{\begin{array}{l}
-\Delta \phi_{2}(\Delta u(t-1))=\lambda \sqrt{1+u}+\arctan (0.005 u) \quad t \in[1,2]_{\mathbb{Z}} \\
u(1)=u(2), \Delta u(1)=\Delta u(2)
\end{array}\right. \\
F(t, \xi)=\int_{0}^{\tau} f(t, u) d x=\int_{0}^{\tau} \sqrt{(1+x)} d x=\frac{(1+\xi)^{\frac{3}{2}}}{\frac{3}{2}} \leq \frac{2}{3}(1+\xi)^{\frac{3}{2}}, \\
\sum_{t=2}^{2} \max _{|\xi| \leq 2.1} F(t, \xi)=7.27
\end{gathered}
$$

By considering $L=0.005, \zeta=0.9, \alpha=0.5, \epsilon=2.1$ and $\beta=3.1$ By using Theorems 5 and 6 , we get that the problem in Equation (18) has at least one nontrivial solution for every $\lambda \in(0,0.414)$ and for all $\lambda \in(0.944,2.411)$ the problem has at least three solutions.

Author Contributions: All the authors contributed equally.

Funding: This work was supported by University of Mazandaran, Islamic university, Najaf and Iraqi Cultural Attaché-Tehran.

Conflicts of Interest: The authors declare no conflict of interest.

\section{References}

1. Agarwal, R.P.; Perera, K.; ÓRegan, D. Multiple positive solutions of singular discrete $p$-Laplacian problems via variational methods. Adv. Differ. Equ. 2005, 2, 93-99. [CrossRef]

2. Anderson, D.R.; Minhòs, F. A discrete fourth order Lidstone system with parameters. Appl. Math. Comput. 2009, 214, 523-533.

3. Bereanu, C.; Jebelean, P.; Mawhin, J. Multiple solutions for Neumann and periodic problems with singular $\phi$-Laplacian. J. Funct. Anal. 2011, 261, 3226-3246. [CrossRef]

4. Mawhin, J. Periodic solutions of second order Lagrangian difference systems with bounded or singular $\phi$-Laplacian and periodic potential. Discret. Contin. Dyn. Syst. 2013, 6, 1065-1076. [CrossRef]

5. Mawhin, J. Periodic solutions of Lagrangian difference systems: Periodic nonlinearities (almost) don't matter. Contemp. Math. 2013, 595, 265-279.

6. Ricceri, B. A general variational principle and some of its applications. J. Comput. Appl. Math. 2000, 113, 401-410. [CrossRef] 
7. Bisci, G.M.; Repovš, D. Existence of solutions for $p$-Laplacian discrete equations. J. Appl. Math. Comput. 2014, 242, 454-461. [CrossRef]

8. Ma, R.; Xu, Y. Existence of positive solution for nonlinear fourth-order difference equations. J. Appl. Math. Comput. 2010, 59, 3770-3777. [CrossRef]

9. Ma, R.; Gao, C. Bifurcation of positive solutions of a nonlinear discrete fourth-order boundary value problem. Z. Angew. Math. Phys. 2013, 64, 493-506. [CrossRef]

10. Anderson, D.R.; Bonanno, G. A three critical points theorem and its applications to the ordinary Dirichlet problem. Topol. Methods Nonlinear Anal. 2003, 22, 93-103.

11. Luo, Z.M.; Zhang, X.Y. Existence of nonconstant periodic solutions for a nonlinear discrete system involving the p-laplacian. Bull. Malays. Math. Sci. Soc. 2012, 35, 373-382.

(C) 2019 by the authors. Licensee MDPI, Basel, Switzerland. This article is an open access article distributed under the terms and conditions of the Creative Commons Attribution (CC BY) license (http:/ / creativecommons.org/licenses/by/4.0/). 